\title{
Graphite formation in diamond-like carbon thin films
}

\author{
V. A. Plotnikov`, B. F. Dem'yanov, V.I. Yartsev, C. V. Solomatin \\ †plotnikov@phys.asu.ru
}

Altai State University, 61 Lenin st., 656049, Barnaul, Russia

In the present work, particularities of the formation and structure of a graphite phase appearing in a thin amorphous diamondlike carbon (ta-C) film were studied using the method of high resolution electron microscopy. In order to obtain a thin carbon film, a neodymium laser NTS300 with a wavelength of $1064 \mathrm{~nm}$ was used. The film was deposited on a glass substrate in the process of a direct evaporation of a graphite target. Study of the graphite phase formation in the film was carried out using the high resolution transmission electron microscopy that allowed for a direct observation of the thin film atomic structure. Electron diffraction analysis shows that in local areas of a thin film a phase transition from amorphous diamond-like ta-C state to graphite occurs. Single crystals of graphite are oriented so that their basic hexagonal planes are parallel to the film surface. This is due to the fact that $\mathrm{sp}^{3}$ bonds are metastable and can be unstable, particularly on the film surface. Therefore, the transition of $\mathrm{sp}^{3}$ bonds to $\mathrm{sp}^{2}$ ones with the formation of planar complexes with a ring structure initially occurs on the film surface. The growth of a graphite crystal nucleus in the ta-C matrix increases the stress and stabilizes the diamond state. Due to this, the graphite phases form dominantly on the film edges. Analysis of electron diffraction patterns has shown that the interplanar spacings depend on the orientation of the planes relative to the film edge. Measurements of interplanar spacings reveals a complicated character of the stress state of the graphite lattice in the thin film. Along with tension-compression stresses, shear stresses are present and they result in a change of the angles between carbon interatomic bonds.

Keywords: carbon film, diamond-like structure, amorphous carbon, graphite.

\section{Образование графита в алмазоподобных тонких углеродных}

\section{пленках}

\author{
Плотников В. А. ${ }^{\dagger}$ Демьянов Б. Ф., Ярцев В.И., Соломатин К.В. \\ †plotnikov@phys.asu.ru
}

Алтайский государственный университет, пр. Ленина 61, 656049, Барнаул, Россия

В работе методом высокоразрешающей электронной микроскопии проведено исследование особенностей образования и структуры графитовой фазы, возникающей в тонкой аморфной алмазоподобной (ta-C) углеродной пленке. Для получения тонкой углеродной пленки использовался мощный неодимовый лазер NTS300 с длиной волны 1064 нм. Углерод осаждался на стеклянной подложке из силикатного стекла в процессе прямого испарения графитовой мишени. Исследование образования графитовой фазы в пленке проводилось методом высокоразрешающей электронной микроскопии, позволяющей использовать прямое наблюдение атомной структуры тонкой пленки. Электронографический анализ показал, что в локальных областях тонкой пленки происходит фазовый переход из аморфного алмазоподобного tа-С состояния в графит. Монокристаллы графита ориентированы так, что базисная гексагональная плоскость параллельна поверхности пленки. Это связано с тем, что связи sр ${ }^{3}$ метастабильны и могут быть неустойчивы, особенно на поверхности пленки. Поэтому переход sр ${ }^{3}$ связей в $\mathrm{sp}^{2}$ связи с образованием плоских комплексов с кольцевой структурой первоначально происходит на поверхности пленки. Рост зародыша графитового кристалла в ta-C матрице увеличивает напряжение и стабилизирует алмазное состояние. Поэтому образование графитовых фаз происходит преимущественно на краях пленки. Анализ электронограмм от кристаллов графита обнаружил зависимость значений межплоскостных расстояний от ориентации плоскостей относительно края пленки. Измерение значений межплоскостных расстояний показал сложный характер напряженного состояния решетки графита в тонкой пленке. Кроме напряжений типа растяжение-сжатие, присутствуют сдвиговые напряжения, приводящие к изменению значений углов между направлениями углеродных межатомных связей.

Ключевые слова: углеродная пленка, алмазоподобная структура, аморфный углерод, графит. 


\section{1. Введение}

Тонкие углеродные пленки вызывают большой интерес как материалы, обладающие многими важными свойствами, такими как высокая химическая инертность, высокая твердость, уникальные оптические, тепловые и трибологические свойства, что дает хорошие перспективы для их промышленного использования. Особенности свойств углеродных пленок можно объяснить характером межатомных связей между атомами углерода [1]. Наиболее распространенные химические связи в углероде определяются $\mathrm{sp}^{2}$ - и $\mathrm{sp}^{3}$ гибридизацией электронов. По преимущественному типу связи углеродные пленки могут быть классифицированы как алмазоподобные (diamond-like carbon) или графитоподобные (graphite-like carbon) [1-4]. Наибольший интерес представляют пленки, имеющие алмазоподобные свойства. При определенных режимах конденсации углерода возможно сформировать аморфные алмазные пленки, состоящие преимущественно из хаотически ориентированных кластеров углерода с тетраэдрическим связями, которые классифицируются в литературе как ta-C (tetrahedral amorphous carbon) пленки [4-7].

В зависимости от режима синтеза доля $\mathrm{sp}^{3}$ связей и, соответственно, свойства алмазоподобных пленок изменяются в широких пределах [8-11]. Образование специфической «островковой» структуры поверхности пленок [8,11] определяет трибологические [12], электрические [13], гидрофобные [14] свойства. Также обнаружено влияние на структуру и свойства тонких пленок материала подложки $[11,15,16]$. Основные достижения и проблемы получения и применения алмазоподобных тонких пленок рассмотрены в обзоре [17].

Кроме того необходимо учитывать, что алмазоподобное состояние является метастабильным и возникает вопрос об устойчивости структуры и сохранении свойств в процессе эксплуатации. Поэтому несмотря на большое количество работ выполненных в последнее время по аморфным алмазоподобным углеродным пленкам имеется необходимость дальнейшего их исследования для определения свойств, структуры и пределов стабильности аморфного состояния.

В настоящей работе проведено исследование особенностей образования и структуры графитовой фазы возникающей в тонкой аморфной алмазоподобной (ta-C) углеродной пленке.

\section{2. Методика эксперимента}

Экспериментальное получение алмазоподобных пленок наиболее эффективно происходит в процессе поатомной сборки, например, при конденсации углерода после лазерного испарения углеродной мишени. В этом случае имеется высокая вероятность формирования структур с максимальным числом тетрагональных групп атомов. Лазерное испарение и последующая конденсация углерода на аморфную подложку из силикатного стекла дает преимущественно $\mathrm{sp}^{3}$ связи, так называемый ta-С углерод.
В нашем случае для получения углеродной пленки применялся способ прямого испарения графитовой мишени при воздействии лазерного излучения с длиной волны 1064 нм неодимового лазера NTS300. Расфокусированный лазерный пучок вводился в вакуумную камеру установки ВУП-4, где располагались графитовые мишени и подложки из силикатного стекла. Полученный поток испаряемого углерода попадал на стеклянную подложку, формируя углеродную пленку. В ходе лазерного нагрева расфокусированным лазерным пучком фрагментация мишени отсутствовала.

Результаты исследования тонкой структуры углеродной пленки [18] показали, что материал пленки состоит из хаотически разориентированных кластеров алмаза с размером не более 1 нм. Пленка имеет однородную структуру, т.е. сопрягающиеся кластеры не разделены границами, одно зерно переходит в другое без скачкообразного изменения структуры. Такой плавный переход структуры одного зерна в структуру другого зерна может быть объяснен аморфным состоянием углеродной пленки и позволяет идентифицировать материал синтезированной углеродной пленки как ta-C аморфный алмаз.

Структурные исследования проводились в лаборатории «ИРГЕТАС» Восточно-Казахстанского государственного технического университета им. Д. Серикбаева, на просвечивающем электронном микроскопе высокого разрешения JEM-2100 при ускоряющем напряжении 200 кВ.

\section{3. Образование графитовой фазы}

Исследование образования графитовой фазы методом высокоразрешающей электронной микроскопии позволяет наблюдать особенности атомной структуры тонкой пленки. На рис. 1 показана область, расположенная вблизи края пленки. Отчетливо видны кристаллографические плоскости, что свидетельствует о присутствии хорошо сформированной кристаллической решетки. Электронограмма, полученная от этого участка, является точечной и показывает наличие крупного монокристалла. Прямое измерение дает размер монокристалла более 40 нм. Окружающие монокристалл области пленки состоят из аморфной фазы. На рис. 1 аморфная фаза выглядит как слабоконтрастная зернистая структура.

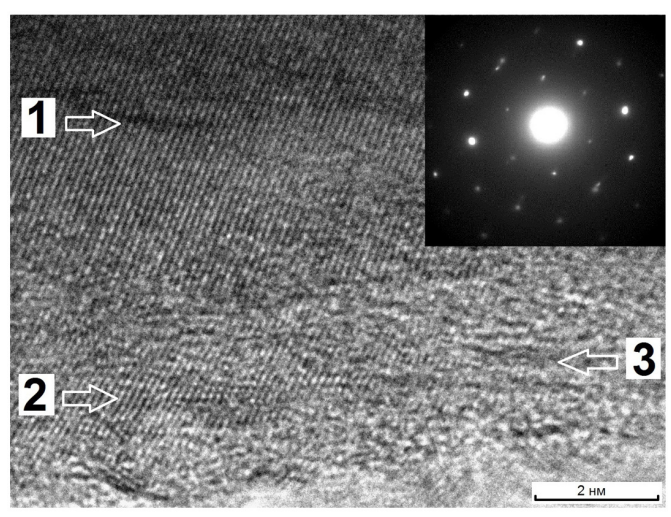

Рис. 1. Фронт кристаллизации графита.

Fig. 1. The front of graphite crystallization. 
Расшифровка электронограммы показала, что углерод имеет гексагональную решетку, то есть монокристалл является графитом. Таким образом, в локальной области тонкой пленки произошел фазовый переход из аморфного алмазоподобного ta-C состояния в графит. Характерно, что монокристалл графита ориентирован так, что базисная гексагональная плоскость параллельна поверхности пленки. Такая ориентация является типичной для графита в исследуемой тонкой пленке. Все обнаруженные кристаллы графита имеют одинаковую ориентацию базисной плоскости параллельно поверхности пленки.

Алмазоподобная структура является метастабильной, но достаточно устойчивой. В вакууме она сохраняется до $1000-1100^{\circ} \mathrm{C}$, на воздухе окисляется при $450-500^{\circ} \mathrm{C}[1]$. Закономерности перехода алмаза в графит зависят от структурного состояния алмаза, то есть находится ли он в нанокристаллическом или аморфном состоянии, образует ли массивный материал или низкоразмерную структуру. Превращение в массивном материале происходит в несколько стадий. На первой стадии образуются гексагональные слои (графитовые плоскости, состоящие из нескольких гексагональных колец), беспорядочно разориентированные друг относительно друга. Это так называемая турбостратная структура графита $[19,20]$. При этом возможно существенное изменение величины межплоскостных расстояний. В дальнейшем происходит увеличение степени взаимной ориентации слоев и формируется графитовое зерно.

В тонких пленках механизм графитизации, по-видимому, имеет существенные отличия. При переходе кластеров алмаза в графит графитовые гексагональные плоскости ориентированы параллельно поверхности пленки. Связи $\mathrm{sp}^{3}$ метастабильны и могут быть неустойчивы особенно на поверхности пленки. Экспериментальные исследования показывают, что в поверхностном слое толщиной 1 нм, доля $\mathrm{sp}^{3}$ связей меньше, чем в центральной части пленки [2]. Кроме того поверхность ta-С пленки стремится к минимуму оборванных связей, поэтому можно ожидать переход $\mathrm{sp}^{3}$ связей в $\mathrm{sp}^{2}$ связи с образованием плоских комплексов с кольцевой структурой. Эти участки графитовой структуры должны уменьшать поверхностную энергию, этому способствует и ориентация плоскостей, гексагональные плоскости параллельны поверхности пленки. Эти рассуждения подтверждаются моделированием [21,22].

Второй фактор, который необходимо учитывать при анализе механизма фазового перехода, это высокие упругие напряжения, существующие в тонких пленках [23]. Переход $\mathrm{sp}^{3}$ связей в $\mathrm{sp}^{2}$ выгоден, так как снижает напряжения в пленке [24]. Это связано с тем, что длина $\mathrm{sp}^{2}$ связи (0,1422 нм) меньше, чем длина $\mathrm{sp}^{3}$ связи (0,154 нм). Высокие напряжения в пленке в целом стабилизируют алмазное состояние [1], а снижение напряжений уменьшает энергетический барьер фазового перехода. Поэтому, образование графитовых колец на поверхности углеродной пленки является энергетически выгодным и следует ожидать появления графитовой структуры в приповерхностном слое.
Образование объемной графитовой фазы, напротив, увеличивает напряжения т. к. плотность образующейся фазы уменьшается, соответственно, увеличивается объем фазы. Плотность алмаза 3,515 г/ $\mathrm{cm}^{3}$, плотность графита 2,267 г/ $\mathrm{cm}^{3}$, плотность аморфного состояния ta-C 3,1 г/см³. Образование зародыша графитового кристалла в ta-C матрице увеличивает напряжение и стабилизирует алмазное состояние. Поэтому, образование объемных, захватывающих всю толщину пленки графитовых фаз следует ожидать преимущественно на краях пленки. Именно на краях пленки обнаружены фазы графита в нашем случае.

Переход в графит начинается с некоторой области. Поскольку превращение в графит является энергетически выгодным, графитовое зерно растет, захватывая окружающие участки углеродной пленки. Кластерная алмазная структура переходит в монокристаллическую графитовую. Этот процесс распространяется постепенно, можно наблюдать фронт превращения. Фронт кристаллизации графита показан на рис. 1. В области 1 графитовая фаза полностью сформирована, видны правильные ряды кристаллографических плоскостей. В области 2 графит имеет не полностью сформированную искаженную решетку, однако, как хорошо видно, ее ориентация совпадает с кристаллом в зоне 1 , в дальнейшем они образуют единый монокристалл. Область 3 представляет аморфную фазу, соответствующую основному материалу тонкой пленки.

\section{4. Структура графита}

На рис. 2 показана структура зерна графита, полученная методом высокоразрешающей электронной микроскопии. Представляет интерес исследовать решетку образующейся графитовой фазы, используя атомное разрешение. На изображении графита хорошо видны не только кристаллографические плоскости, но и положения отдельных атомов. Расшифровка электронограммы, приведенной на рис. 2, показала, что графит ориентирован таким образом, что его базисная плоскость параллельна плоскости пленки. Увеличенная атомная структура графита с выделением шестиугольного базисного элемента приведена в правом верхнем углу. Видно, что структура шестиугольных колец сильно искажена. Деформация колец приводит к тому, что значения межплоскостного расстояния $d_{100}$ зависят от ориентации плоскости (100) относительно края пленки. Расчеты показали следующие значения для трех различно ориентированных систем плоскостей

$$
\mathrm{d}_{100}^{1}=0,198 \mathrm{HM}, \mathrm{d}_{100}^{2}=0,210 \mathrm{HM}, \mathrm{d}_{100}^{3}=0,247 \text { нм. }
$$

В массивном графите $\mathrm{d}_{100}=0,213$ нм.

Деформация графитовых колец приводит к изменению угла между направлениями углеродных межатомных связей. Равновесное значение угла составляет $120^{\circ}$, в тонкой же пленке углы имеют следующие значения: $107^{\circ}, 120^{\circ}$ и $133^{\circ}$. Такие изменения величин углов и межплоскостных расстояний показывают не только наличие искажения решетки типа растяжение-сжатие, но и присутствие сдвиговой деформации. 


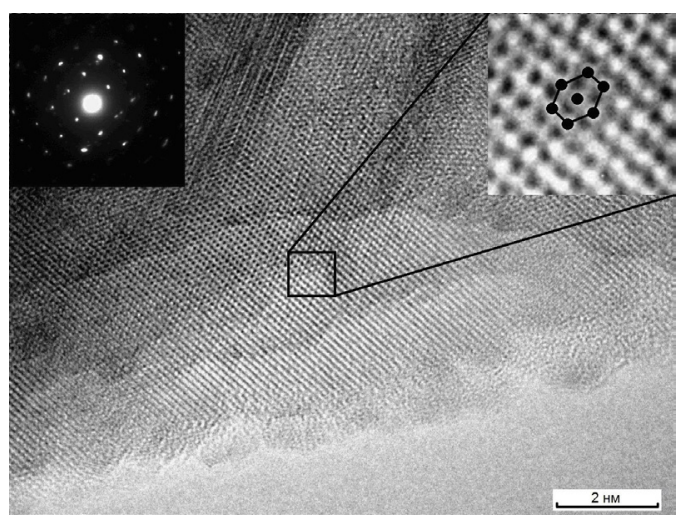

Рис. 2. Искаженная структура базисной плоскости графита. Fig. 2. The distorted structure of the basic plane of graphite.

Структуру кристаллических областей графита с отличающимися межплоскостными расстояниями нельзя считать дефектной. В частности, прямые наблюдения показали отсутствие дислокаций. Следовательно, искажение межатомных связей может быть связано с особым состоянием решетки в тонкой пленке. Одним из факторов может быть присутствие высоких напряжений, приводящих к изменению угловых направлений связей. На рис. 3 показан фрагмент базисной плоскости графита. Отмечены межплоскостные расстояния $d_{100}$ и расстояния $R_{c-c}$ между атомами. Видно, что межплоскостные расстояния напрямую связаны с угловыми характеристиками межатомных связей. Направленность связей менее жесткая характеристика, чем межатомное расстояние. Достаточно вспомнить структуру углеродных нанотрубок и фуллеренов, формирование которых во многом связано с изменением углового направления связей [25].

Второй фактор - изменение межатомных расстояний за счет малого размера образующегося кристалла графита. Один из размеров кристалла определяется толщиной пленки, которая может составлять несколько нм. Ранее в ряде работ показано, что существует зависимость между размерами кристалла графита и значениями межплоскостных расстояний [26]. Причиной изменения межплоскостных расстояний в низкоразмерных углеродных материалах является уменьшение межатомных расстояний $R_{c-c}$ связанное с изменением кратности связей. В тонких пленках доля поверхностного состояния атомов и, следовательно, незамкнутых $\pi$-связей возрастает, $\pi$-электроны участвуют в образовании $\sigma$-связей, что увеличивает кратность ковалентной $\sigma$-связи и ее укорочение [20,27].

В недавней работе [28] методами компьютерного моделирования проведено исследование влияния толщины ta-C пленки на ее структуру. Показано, что при толщинах пленки 2-6 нм происходит изменение не только длины межатомных связей, но и угловых направлений. Таким образом, в тонких пленках геометрические параметры ковалентных связей не являются строго фиксированными, а могут изменяться в достаточно большом диапазоне значений. Этот эффект может быть существенным, если учесть присутствие в пленке упругих напряжений.

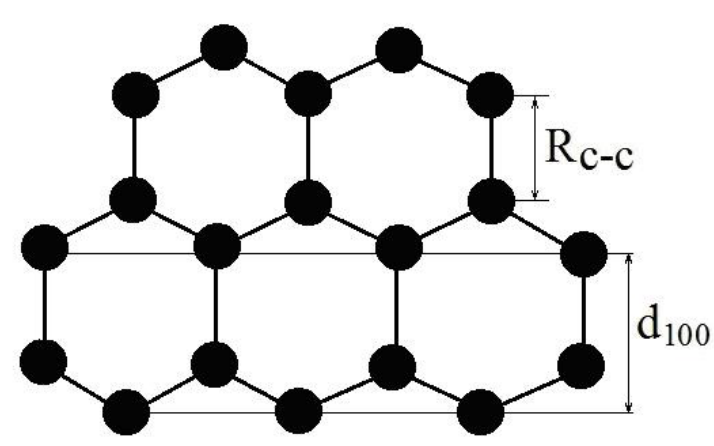

Рис. 3. Гексагональные кольца, образующие базисные плоскости графита.

Fig. 3. The hexagonal rings, forming the basic planes of graphite.

\section{5. Заключение}

Проведенные исследования образования графитовой фазы в тонкой аморфной алмазоподобной (ta-C) углеродной пленке показали существование ряда особенностей, характерных для тонких пленок. При переходе кластеров алмаза в графит графитовые гексагональные плоскости ориентированы параллельно поверхности пленки. Упругие напряжения в тонкой углеродной пленке стабилизируют алмазную фазу, поэтому образование объемной графитовой фазы происходит вблизи края пленки. Структура шестиугольных колец графита искажена, что приводит к изменению угла между направлениями углеродных межатомных связей. Характер искажений показывает присутствие как напряжений типа растяжение-сжатие, так и сдвиговых напряжений.

\section{Литература/References}

1. J. Robertson. Mater. Sci. Eng. R 37, 129-281 (2002).

2. M.G. Beghi, A.C. Ferrari, K.B. K. Teo, J. Robertson, C.E. Bottani, A. Libassi, B.K. Tanner. Appl. Phys. Lett. 81, 3804-3806 (2002).

3. P. Zhang, B. K. Tay, C. Q. Sun, S. P. Lau. J. Vac. Sci. Technol. A 20, 1390-1394 (2002).

4. B. K. Tay, D. Sheeja, S. P. Lau, X. Shi, B. C. Seet, Y. C. Yeo. Surf. Coat. Technol. 130, 248-251 (2000).

5. K. W. R. Gilkes, P.H. Gaskell, J. Robertson. Phys. Rev. B. 51, 12303- 12312 (1995).

6. Y. Lifshitz. Diamond Relat. Mater. 8, 1659 - 1676 (1999).

7. S. Xu, B. K. Tay, H. S. Tan, L. Zhong, Y. Q. Tu, S. R. P. Silva, W. I. Milne. J. Appl. Phys. 79, 7234 - 7240 (1996).

8. R. Maheswaran, S. Ramaswamy, D. J. Thiruvadigal, C. Gopalakrishnan.J. Non-Crystalline Solids. 357, $1710-1715$ (2011).

9. A. Sikora, F. Garrelie, C. Donnet, A.S. Loir, J. Fontaine, J.C. Sanchez-Lopez, T.C. Rojas. J. Appl. Phys. 108, 113516 (2010).

10. D.S. Lisovenko, J.A. Baimova, L. Kh. Rysaeva, V. A. Gorodtsov, A.I. Rudskoy, S.V. Dmitriev. Physica status solidi (b). 253, 1295 - 1302 (2016).

11. N. Dwivedi, S. Kumar, H.K. Malik. J. Appl. Phys. 112, 023518 (2012). 
12. S. V. Hainsworth, N.J. Uhure. International Materials Reviews, 52, 153 - 174 (2007).

13. A. Sikora, P. Paolino, H. Ftouni, C. Guerret-Piécourt, J.-L. Garden, A.-S. Loir, F. Garrelie, C. Donnet, O. Bourgeois. Appl. Phys. Lett. 96, 162111 (2010).

14. A. M. Asl, P. Kameli, M. Ranjbar, H. Salamati, M. Jannesari. Superlattices and Microstructures. 81, 64-79 (2015).

15. D. He, S. Zheng, J. Pu, G. Zhang, L.Hu. Tribology International A. 82, 20-27 (2015).

16. S. A. Hevia, F. Guzman-Olivos, I. Munoz, G. MunozCordovez, S. Caballero-Bendixsen, H. M. Ruiz, M. Favre. Surf. Coat. Technol. 312, 55-60 (2017)

17. K. Bewilogua, D. Hofmann. Surf. Coat. Technol. 242, 214-225 (2014).

18. B.F. Dem'yanov, V.A. Plotnikov, V.I. Yartsev, C. V. Solomatin. BPMS. 12 (4), 437-443 (2016) (in Russian) [Б. Ф. Демьянов, В. А. Плотников, В. И. Ярцев, К. В. Соломатин. ФПСМ. 12 (4), 437 - 443 (2016)]

19. Островский В.С., Виргильев Ю.С., Костиков В.И., Шипков Н.Н. Искусственный графит. М.: Металлургия, 1986, 272 с.

20. Шулепов С.В. Физика углеграфитовых материалов. Челябинск: Металлургия. 1990. 336 с.

21. J. Dong, D. A. Drabold. Phys. Rev. B. 57, $15591-15598$
(1998).

22. P. Kelires. J. Non-Crystal. Solids. 227 - 230, (1), 597 - 601 (1998).

23. V.I. Yartsev, B.F. Demyanov, V.A. Plotnikov, S. V. Makarov, C. V. Solomatin. BPMS. 12 (4), 477-481 (2015) (in Russian) [В.И. Ярцев, Б.Ф. Демьянов, В.А. Плотников, С.В. Макаров, К.В. Соломатин. ФПСМ. 12 (4), 477 - 481 (2015)]

24. J. P Sullivan, T. A. Friedmann, A. G. Baca. J. Electr. Mater. 26, 1021 - 1029 (1997).

25. M. D.Starostenkov, I. V.Loshchina, B. F.Dem'yanov. BPMS. 2 (1), 62 -67 (2005) (in Russian) [М.Д. Старостенков, И. В. Лощина, Б. Ф. Демьянов. ФПСМ. 2 (1), 62 -67 (2005)]

26. E. A. Belenkov, A. I. Sheinkman. Russian Physics Journal. 34 (10), 903 - 905 (1992) (in Russian) [Е.А. Беленков, А.И. Шейнкман. Известия вузов. Физика. №10. С. $67-69.1991]$

27. E. A. Belenkov, E.A. Karnaukhov. Physics of the Solid State. 41 (4), 672-675 (1999) (in Russian) [Е.А. Беленков, Е.А. Карнаухов. Физика твердого тела. 41 (4), 744 - 747 (1999)]

28. X. Li, S. Xu, P. Ke, A. Wang. Surf. Coat. Technol. 258, $938-942$ (2014). 